\title{
ONTOLOGY FOR MODELING AND SIMULATION
}

\author{
Charles Turnitsa \\ Jose J. Padilla \\ Old Dominion University \\ Virginia Modeling, Analysis \& Simulation Center \\ Norfolk, VA 23435
}

\author{
Andreas Tolk \\ Old Dominion University \\ Engineering Management \& Systems Engineering \\ Norfolk, VA 23529
}

\begin{abstract}
This paper establishes what makes an ontology different in Modeling and Simulation (M\&S) from other disciplines, vis-a-vis, the necessity to capture a conceptual model of a system in an explicit, unambiguous, and machine readable form. Unlike other disciplines where ontologies are used, such as Information Systems and Medicine, ontologies in M\&S do not depart from a set of requirements but from a research question which is contingent on a modeler. Thus, the semiotic triangle is used to present that different implemented ontologies are representations of different conceptual models whose commonality depends on which research question is being asked. Ontologies can be applied to better capture the modeler's perspective. The elicitation of ontological, epistemological, and teleological considerations is suggested. These considerations may lead to better differentiation between conceptualizations, which for a computer are of importance for use, reuse and composability of models and interoperability of simulations.
\end{abstract}

\section{INTRODUCTION}

Traditionally, an ontological representation, also known as an ontology, is a specification of a system in reality. One of the most commonly referenced definitions for what an ontological representation is, with regards to information systems (vs. philosophy) is "formal explicit specification of a shared conceptualization" (Gruber, 1993). While a conceptualization, in this definition, is behind nearly any artifact (whether it be a computer program, a model, or just a diagram), the specification can vary widely with the intended use it will serve. In addition, the idea of a shared conceptualization assumes a common frame of reference, or lens, which can vary depending on the modeler.

The uses of an ontology have been shown to fall into a framework (Uschold and Jasper 1999) with four categories. These categories have been shown to apply to M\&S usage as well (Turnitsa and Tolk 2006). These four framework categories are:

- Ontology-based search - This is useful for discovery and selection of information or components.

- Neutral authoring - Especially helpful in cases of data exchange among systems.

- Ontology as specification - Useful for describing the meaning of a domain, to guide system development.

- Common access to information - A much stronger case than Neutral Authoring, this allows meaning to be transmitted, not just matched against.

Ontologies in the first and second category are intended to assist automata in performing tasks of search and selection and tasks of meaningful information exchange respectively. Ontologies in the third 
and fourth category will result in artifacts that are primarily intended for human consumption; in specification for development for the former, and definitional knowledge based systems for the latter. However, all four cases are employed within the community to exhibit semantic meaning of what is occurring in an implemented simulation or simulation component, not in the conceptual model (CM) as the $\mathrm{CM}$ has been recognized as the integral part of the M\&S application development (Balci and Ormsby 2007). Focus on implemented simulations can be observed, for instance, in the cases of the Discrete Event Modeling Ontology (DeMO) and Component Oriented Simulation and Modeling Ontology (COSMO).

In a reported successful application of DeMO (Silver, Hassan, and Miller 2007), the ontology was used in a combined application of categories of ontology-based search, and neutral authoring. It was used to assist with the identification and connection of elements from a medical ontological representation, and then to assist with bringing those together with a discrete event system. In both cases, the artifact of the medical ontological representation and the discrete event system were already implemented systems. Although these systems were developed based on conceptual models, those models were not what the DeMO application connected. COSMO is used in the CODES project (Teo and Szabo 2007) in the role of ontology based search. It assists with the location of components that have a semantic match to what is required for Composable Discrete Event Simulation. Again, it is, properly for its role, being employed for the location and selection of implemented components, so it is describing their functional utility, not the modeling decisions and assumptions that went into their design.

This differentiation between what is modeling oriented and what is implementation oriented, as in the previous examples, is important for M\&S.

Modeling and Simulation comprises two parts: while modeling resides on the abstraction level, simulation resides on the implementation level. In modeling, we answer the question what we model, in simulation we answer the question how we model. In order to be able to model, we furthermore need to know why we model (modelers intent), which is defined by requirements derived from the experimental frame or the context of the model, or in academic research the research question. In M\&S as in other disciplines, ontologies need to capture reality in a form that is computer understandable. However, in $M \& S$ this reality is contingent on the modeler and on a research question. In other words: the focus on this contribution lies on ontological support on formulating the research questions and the modeling, not focusing on simulation.

This paper is organized as follows: section two makes the case why ontologies in M\&S should be treated differently than in other disciplines. Section 3 makes recommendations of the considerations that modelers need to be aware of when going through the modeling process and that should be captured in the conceptual model. Section 4 presents the relevance of the presented considerations on composability and interoperability. Finally, section 5 present conclusions and final remarks.

\section{ONTOLOGIES IN MODELING AND SIMULATION}

First and foremost, the authors take a view similar to Raubal and Kuhn (2004) that differentiates between ontology as the domain of philosophy dealing with the appreciation of existence, from the domain of system representation that deals with codifying the contents and knowledge of a system. This paper concerns itself with the latter, as seems appropriate for research in the fields of information technology, modeling and simulation, and systems engineering. This distinction also follows from Guarino (1998), where it is suggested that the difference be made clear by the term "Ontology" (significance intentional) for philosophy, and "ontologies" for methods of capturing system knowledge. Here we will use the phrase ontological representation, or an ontology (ontologies is the plural term from an ontology. Ontology, as the study of reality, does not have a plural term), to stand for that second meaning.

\subsection{Different Descriptions of Reality: The Semiotic Triangle}

In the pursuit of linguistics and the study of the elements of language and their meaning, there is an idea that comes to us known as the semiotic triangle. Presented by Ogden and Richards (1923), the idea comes 


\section{Turnitsa, Padilla and Tolk}

from a series of similar ideas through history. Aristotle differentiated objects from the words that refer to them and the ideal types that we consider them as. Frege had the Bedeutung for the object (or referent), the Sinn for the concept, and the Zeichen for the symbol representing that concept. A treatment of these ideas is in Sowa (2000). What is common is the differentiation of what is real, observed, or assumed, how it is perceived and modeled, and how it is represented.

The idea of the semiotic triangle is, briefly, that when one wishes to communicate about some referent, one first has awareness of the referent. Then a conceptualization of that referent is formed, and finally some symbol (a word, a picture) is selected or formed that stands for that conceptualization. These are the three vertices of the triangle - referent, conceptualization, and symbol. The connecting edges can be considered as (1) if the conceptualization is adequate to capture the referent, (2) if the symbol is a correct choice to representing the conceptualization, and (3) if the symbol then is perceived as being a proxy for the referent. Although we assume to communicate about the referent, we are actually communicating about our conceptualization thereof, using symbols.

The applicability to modeling is that a model is a conceptualization of a referent, and a simulation is the expression of that conceptualization into a decidable system. Even more so, the conceptualization becomes the reality of the simulation: the referent is no longer accessible, the symbols, tags, or labels, together with implemented structures and capabilities, are what is used to answer the research question. Formally, this consideration of the traversal of the triangle is a representation of the entire referent-modelsimulation process, and helps to identify the difference between the process of eliciting a model, and then choosing a simulation to represent that model (differentiating between the two paradigms). By considering either of the second or third nodes in isolation, it can be seen how the triangle applies in almost a fractal sense. By looking at the idea of forming a model, once again the referent becomes the original node, and then the conceptualization of what about the referent is germane to the model becomes the second node, and finally the output (or symbol) is the model itself. Equally, the same process can be applied to the sequence of activity of proceeding from a model to a simulation.

As the steps of conceptualizing, and then choosing a symbol to represent that conceptualization are cognitive acts, the perspective and reasons for doing so vary from individual to individual. Because of this, we can see the implications that there are an uncountable number of possible conceptualizations arising from one referent, and an equally uncountable number of possible symbols that can represent one conceptualization. When this is applied to the paradigms of modeling and simulation, it becomes clear how one referent can be represented (based on the goal of the actor doing the representing, also known as "modeler's intent") as a near infinite number of different models, and how each model could be represented by a near infinite number of simulations.

An ontological representation of a system is an explicit specification of a conceptualization (Gruber 1993). Considering the knowledge behind a system as a conceptualization matches the application made of the semiotic triangle in representing how some idea gets from referent to symbol (through conceptualization). By extension, from our impressments of the semiotic triangle to the field of modeling and simulation, we can see that the act of modeling a referent results in some conceptualization, and that by applying Gruber (1993) an ontology representation is generally the means of capturing such a specification. In this regard, a conceptual model can be said to be an ontological representation of the simulation that implements it.

\subsection{The Research Question}

Whereas in most disciplines the M\&S effort focuses on the implementation of a model to replicate an observed behavior, in M\&S as a discipline much of the effort is placed in the modeling aspect and how to capture the system to answer a research question. The emphasis on modeling seeks to "bound" the modeler's intent by limiting the model to one that answers a research question. This difference is better observed in the example provided by Tolk et al. (2010):

"An Automatic Teller Machine (ATM) is representative of a real teller as it can perform many similar interactions including necessary inputs and outputs. In M\&S systems, interactions in terms of inputs and 
outputs are not sufficient to identify a referent because many referents have similar inputs and outputs when abstracted, which makes it impossible to identify which one the conceptualization is referring to. A conceptual model of a teller designed to study the average processing time of a customer is different from an ATM. In this case, the customers and the teller may be abstracted into probability density functions and a queuing system, as this maybe sufficient for the study. The validity of answers is therefore highly dependent on the context of the model. In other words, the conceptual model in M\&S needs to capture data in the form of inputs and outputs, processes that consume the data and needs a way to distinguish conceptualizations of referents from one another by capturing the assumptions and constraints inherent to the model".

As can be seen from this excerpt, there is a suggested differentiation on the need of capturing an ATM's behavior to describe it versus capturing an ATM's behavior with the purpose of answering a research question. The question, in this case, refers to studying the average processing time. The same concept applies when it comes to ontologies; an ontology in M\&S is built to answer or help answering a particular research question. This is a goal that goes beyond the traditional description of reality with the purpose of capturing what is known about a particular system.

It is worth to point out that the ideas presented in this section distinguish M\&S from other software engineering (SWE) disciplines: while in traditional SWE the product supports referents (e.g., software communication between the real ATM and the real bank account), in M\&S the model of ATM, bank, and communication as a conceptualization and its implementation becomes the product. That is what motivates the emphasis on ontological support for capturing the conceptualization in machine readable form to allow reuse, composition, and application in system-of-system contexts. To better understand how to better support the conceptualization process, we distinguish between ontological, epistemological, and teleological aspects in the next section.

\section{ONTOLOGY, EPISTEMOLOGY, AND TELEOLOGY IN M\&S}

As has been suggested, ontologies are needed in M\&S because they are formal specifications that are ultimately computer implementable. This means that they are not only explicit and purposeful abstractions of reality (model), but also provide the added bonus of supporting simulations. However, ontologies' abstraction of modeling process is much overlooked by a greater focus on computer implementations of a perceived reality. This assertion comes with the assumption of an objectively observable reality which is an ontological assumption that needs to be considered. Further, what is represented in an ontology is not solely a perspective on reality but also what is known about reality which is an epistemological assertion that needs to consider how that knowledge came to be. As if the ontological and epistemological considerations were not enough, ontologies are built with a purpose of describing reality or answering a research questions which are teleological considerations. Being the perception of reality contingent on ontological, epistemological, and teleological predispositions or considerations, their awareness during the conceptual modeling of a system is important not only for helping establish the modeler's intent but also for urging their capture in the model. In other words, an ontology comes with preconceptions, beliefs and assumptions of modelers that should be captured in an explicit manner in the modeling process.

\subsection{Ontology}

Ontology is the study of being or the study of what exists. According to Kienzle (1970), the concern of ontology is with reality: what are things made of, how many different kinds of things are there, what is the relation between mind and matter? According to Feibleman (1953):

"Ontology is the widest system in any finite set of systems. It would perforce have to be an abstract body of knowledge and make the claim to truth. This could be either a tentative or an absolute claim. Its own terms of description are the categories of traditional metaphysics. The definition of Bentham, that the field of ontology is the field of supremely abstract entities, refers to these categories; in modern logical 


\section{Turnitsa, Padilla and Tolk}

and mathematical systems we would call these categories the undefined terms employed in the unproved propositions which constitute the postulates of the system. There is no official ontology, and contending ontologies must support their claims on the basis of the same criteria used by other kinds of system: consistency, completeness, and applicability. Rival ontologies exist theoretically and practically, and assert both abstractly and concretely their respective claims."

From this excerpt it can be observed that ontology is the description of reality. However, given that in post-positivistic science views reality is understood as unknowable and indescribable, exemplified by the case of the supreme abstract entities, we have access to different realities which are described under different ontologies. The wider the ontology, the closer the ontology is to be to reality and a claim made in this ontology, is going to be considered an absolute claim. Although each ontology for each model must be in itself consistent, we cannot assume that all model ontologies describing the same referent also are derived from one common ontology that describes one reality. They may differ substantially, in particular in soft science (but, as we know, also in micro and macro scale of physical sciences).

Ontologically, we perceive in substantive or process terms (Rescher 2000). Substantive ontology focuses on describing what something is; in terms of its parts and relations among parts. Process ontology focuses on describing how something is done. However, we describe processes in substantive terms. This can be explained from the perspective that the only way of observing how something is done is by observing the states of how something is transformed. Yet, this transformation is still a description of the thing itself and not on the undergone process.

\subsection{Epistemology}

Epistemology is the study of how we come to know. Epistemological beliefs are individual's belief about the definition of knowledge, how knowledge is constructed, how knowledge is evaluated, where knowledge resides, how knowledge occurs (Hofer 2002). Epistemology seeks to answer the question: what can be considered knowledge? The answer to this question, formulated by the ancient Greeks, is still in debate today with a no clear consensus in sight. Epistemologically, a satisfactory definition of knowledge is that of justified true belief "with conditions." These conditions remark where justified true belief cannot be considered knowledge, such as the Gettier case, where a justified true belief is subject to luck. Pragmatically, epistemology focuses on the validity of knowledge, considering, its sources, how it is justified, and under what conditions claims can truly be considered knowledge.

Epistemologically, we come to know through empirical or rational means. Empirically, we come to know through correspondence; what an individual perceives through his/her senses and it can be proven scientifically or accepted through pragmatical means is accepted as knowledge. Rationally, we come to know through coherence; what an individual creates in his/her mind, whether or not initiated by observation, and can be explained within a system of premises is accepted as knowledge. Both currents are accepted in the body of knowledge and both have supporters and detractors. Biologists and experimental physicists, for instance, abide by seeking knowledge through empirical means. Mathematicians and M\&S researchers, on the other hand, abide by seeking knowledge through rational means. For the conceptualization of a model, the epistemological constraints can therefore easily become very relevant when it comes to identification and selection of composable solutions.

\subsection{Teleology}

Teleology is the study of action and purpose. According to Stacey, Griffin, and Shaw (2000, p. 14), "human action is purposeful and it is important to make clear in one's explanation just how one thinks about that purpose." Currently, the term teleology has a combination of its two main contexts; vis a vis, a purposeful behavior while seeking of a goal. This perspective has been studied in its logical form by philosophers of science. Kernohan (1987), being one of them, posits that in its simplest form, a teleological law looks like: "for any system $x, x$ does $\boldsymbol{B}$ because $x$ is such that $x$ doing $\boldsymbol{B}$, leads to $x$ getting G." However, although intuitively formulated, Kernohan throughout its paper is not capable of defining a 
teleological law without reaching a case where the law would not work. This impossibility stems, among other things, from the infinite recursion needed to explain this type of clause using formal logic, from contradictions when this recursion need to be bypassed, or from counterexamples that negate the conclusion.

Stacey, Griffin, and Shaw (2000, p. 49) propose five different notions of teleology:

- Natural Law Teleology, in which the concepts of self-organization and emergence do not feature at all and there is no change, other than movement to the perfect.

- Rationalist Teleology, which also has no particular implications for self-organization and change is the consequence of human choice.

- Formative Teleology, which implies a form of self-organization that reproduces forms without any significant transformation.

- Transformative Teleology, which implies a form of self-organization as paradoxical, characterized by both continuity and potential radical transformation.

- Adaptionist Teleology, which implies a chance-based competitive search for optimality with a weak form of self-organization confined to the selection process. Change is movement to a stable state of adaptation to the environment.

Through these notions, Stacey, Griffin, and Shaw present a case for self-organization and emergence as criteria for the complexity of an organization. These five notions are found along two extremes: one of a knowable future state and another of an un-knowable future state. These extremes are consistent with Rosenblueth, Wiener, and Bigelow (1943) that see teleology as based on feed-back to be predictive (extrapolative) and non-predictive (non-extrapolative). As with epistemology, teleological constraints can become pivotal for composability.

\subsection{Ontology, Epistemology, and Teleology within the Semiotic Triangle}

The idea of epistemological, ontological and teleological preconceptions or worldviews has been studied from the perspective of problem solving (Bozkurt, Padilla, and Sousa-Poza 2007). Like in this case, they argue that ontology, epistemology, and teleology help establish the embedded worldviews of an individual. Each individual will have a different way of seeing reality, seeking knowledge and establishing purpose. Reality may be absolute, but depending on the lenses an individual uses, reality will be seen and interpreted differently. In the case here presented, these three considerations affect the way an ontology is created, used, or reused given that they contain elements that should be made explicit by the modeler. Unlike Bozkurt, Padilla, and Sousa-Poza (2007), orthogonality cannot be assumed given that these three considerations are highly likely affecting one another and therefore the resulting ontologies. For instance, ontologies are scientific tools that abide under different epistemologies: categorization can be made of observed phenomena or of created phenomena which are consistent with empirical or rationalist epistemologies. Through canons of research, each epistemology now provides truth conditions for the captured and described reality. Ontologies also abide by teleological considerations such as those regarding the purpose of capturing reality, answering a research question, or allowing reasoning capabilities in the implementation.

As of now, the focus on the semiotic triangle has been on the referent-model relation given that this is where ontology and ontologies live. Implementation, the relation model to simulation, is the process of creating an experimental setting through simulation. In this case, the results of that simulation tell us something about the referent without using the referent. At this point, the execution of that model over time would provide and output that can be fed back to the ontological representation in order to improve the description of reality by answering questions about reality. This new knowledge created through rational means can now be included in an ontology that captures a created reality and tells us something about a possible future. 


\section{Turnitsa, Padilla and Tolk}

Placing ontological, epistemological, and teleological considerations along the semiotic triangle, a characterization stands thus:

- Ontological: referent and model nodes in the triangle, answering what is and what describes it. In referent, the ontological perspective of reality; in concept, the ontological representation of reality.

- Epistemological: between nodes of the semiotic triangle via correspondence or coherence, answering what is considered knowledge. In the conceptualization process answering what is true/false about the system; in implementation answering what is true/false about the concept; in the comparison symbol-system answering what is true/false in the symbol that is true/false in the system.

- Teleological: model and simulation nodes with respect to the referent, answering what is the purpose of the model and simulation. In model, what is the driving question of the model; in simulation, what is the driving question of the simulation.

A conceptual model that is intended to support the identification of potential models in support of answering a research question, the selection of the best alternatives, the composition of these models into a coherent system-of-system, and the orchestration of the execution needs to take all these aspects into account.

\section{RELEVANCE TO COMPOSABILITY AND INTEROPERABILITY}

Ontological, epistemological, and teleological considerations during the modeling process affect use, reuse, and composability of models and interoperability of simulations. Composability of models, like interoperability of simulations, is not a condition of models; it is a condition that depends on the modeling question. As such, there may be several ontologies that captured a perspective of reality or captured a theory that were designed to answer a research question. This means that the consideration of a common research question is a necessary but not sufficient condition for composition in that more likely there is not a common point of departure. To at least assess commonalty at a high level, awareness of the three mentioned considerations is needed.

Ontologically, ontologies focus on categorizing what something is and what something does. In other words, ontologies capture a system in a substantive manner. As of now, there is not known ontology of processes in a manner that they capture their how. To capture a how, in an ontological representation, would imply to capture a function and functions cannot be captured in atomic sentences. This differentiation is important given it presents that ontologies are limited to capturing a modeler's reality in a substantive manner and that any process claim refers to a discretization of a process and not to the process itself. Further and as important, ontologies are the first means of identifying either assumption: of an objectively observable reality or a reality subsumed to the modeler. In either case, arrangements to make explicit a particular assumption must be made.

Epistemologically, ontologies have, by definition, an empirical leaning given that they are focused on capturing and describing an observable reality. However, they could also capture models which already

are conceptualizations. In this sense, ontologies are conceptualizations of created realities. These "realities" are human constructs used as a surrogate to a system in reality for which observations may not be possible and a structure may not be clearly identifiable. This is possible, for instance, when theories are captured as models and these models are assembled in a coherent system of premises using ontologies. In this case, the resulting final ontological representation may be a theory itself or a means to create new theory out of existing theories.

Finally, teleologically, ontologies, being purposeful creations to either describe a system or help answering a research question, also have a teleological component mostly assumed to be of a knowable future state. Even through reasoning means, ontologies are bound to epistemic deductive means to live in 
a knowable future state and as such are limited to that extreme. Yet, they do have a purpose while seeking a goal and this purpose guides the decision making process of what to finally convey in the ontology. It is important that teleological claims are highly associated with the modeler's intent or purpose upon which the CM was created. This intent, as suggested, is highly intertwined with ontological and epistemological considerations.

However, ontological, epistemological, and teleological considerations need to be assessed and captured to be of use for reuse and composability. As of now, metadata or upper ontology forms are possible candidate to conducting this task.

\section{CONCLUSIONS AND FINAL REMARKS}

This paper makes the case of the modeling of ontologies in M\&S. It is presented that unlike other disciplines where ontologies are used to describe an assumed objectively observable system, in $M \& S$ ontologies:

- Capture a system subsumed to a modeler's reality which implies many models all equally correct which may be paired with many simulations.

- Capture a system from reality according to a research question which bounds the scope and resolution of models.

- Can capture a created reality from established theories or ideas from a creative process.

- Are forms of conceptual models that are formal enough to be computer implemented.

- Need to capture ontological, epistemological, and teleological considerations for further composability and interoperability.

As ontologies are representations of specifications, focus on how these representations are differentiable by computers need to be considered. Given that they are also abstractions, a computer unlike humans, may not differentiate a specification of a model of a boat than that of a car. This differentiation is extremely important for composability and interoperability as when models and/or simulations are put together they must share context. A model of a car is not interchangeable with that of a boat despite having similar implementations. One step to consider a shared context is that of establishing a common research question. Another possible step is to bound the research question under ontological, epistemological, and teleological considerations especially in a form that model A can be differentiated from model B by a computer. Although the latter is presented as a suggestion, the former is key when seeking composability and interoperability.

The focus of ontological application and research so far focused - although not exclusively - on simulation aspects. With this paper, we make the case to put more emphasis on the modeling aspect of $\mathrm{M} \& \mathrm{~S}$, as the modeling aspects is what distinguishes $\mathrm{M} \& \mathrm{~S}$ engineering from traditional SWE.

\section{REFERENCES}

Bozkurt, I., J. J. Padilla, A. Sousa-Poza. 2007. Philosophical Profile of the Individual, In Proceedings of the 19th IEEE International Engineering Management Conference (IEMC).

Feibleman J. 1953. Ontology. Baltimore, MD: Johns Hopkins Press, 1953.

Gruber, T. 1993. A Translation Approach to Portable Ontology Specifications, Knowledge Acquisition 5(2):199-220.

Guarino, N. 1998. Formal Ontology and information Systems, Published in N. Guarino, ed., Formal Ontology in Information Systems. IOS Press, Trento, Italy.

Kienzle H. J. 1970. Epistemology and Sociology. British Journal of Sociology 21(4):413-424.

Ogden C. K. and I. A. Richards. 1923. The Meaning of Meaning: A Study of the Influence of Language Upon Thought and of the Science of Symbolism. London: Routledge \& Kegan Paul. 
Balci, O., and W. F. Ormsby. 2007. Conceptual modeling for designing large-scale simulations. Journal of Simulation 1(3):175-186.

Raubal M. and W. Kuhn. 2004. Ontology-Based Task Simulation. Spatial Cognition and Computation 4( 1):15-37.

Rescher N. 2000. Process Philosophy: A Survey of Basic Issues. Pittsburgh, PA: University of Pittsburgh Press.

Rosenblueth, A, N. Wiener, and J. Bigelow. 1943. Behavior, Purpose and Teleology. Philosophy of Science . 10(1):18-24.

Silver, G. A., O. Hassan, and J.A. Miller. 2007. From Domain Ontologies to Modeling Ontologies to Executable Simulation Models. In Proceedings of the 2007 Winter Simulation Conference. ed. S.G. Henderson, B. Biller, M.-H. Hsieh,. J. Shortle, J.D.Tew, and R.R. Barton. 1108-1117. Piscataway, New Jersey: Institute of Electrical and Electronics Engineers, Inc.

Stacey, R. D., D. Griffin, and P. Shaw. 2000. Complexity and Management: Fad or Radical Challenge to Systems Thinking? Routledge, Taylor and Francis Group.

Sowa, J. F. 2000. Ontology, Metadata, and Semiotics. Presented at ICCS'2000 in Darmstadt, Germany, on August 14, 2000. Published in B. Ganter \& G. W. Mineau, eds., Conceptual Structures: Logical, Linguistic, and Computational Issues, Lecture Notes in AI \#1867, Springer-Verlag, Berlin.

Teo, Y. M. and C. Szabo. 2007. CODES: An Integrated Approach to Composable Modeling and Simulation. Technical Report APSTC-TR-2007-04, Asian Pacific Science and Technology Center.

Tolk, A., S. Y. Diallo, R. D. King, J. J. Padilla and C. D. Turnitsa. 2010. Conceptual Modeling for Composition of Model-based Complex Systems. In Conceptual Modelling for Discrete-Event Simulation, ed. S. Robinson, R. Brooks, K. Kotiadis and D.-J. van der Zee, CRC Press.

Turnitsa, C. and A. Tolk. 2006. Ontology Applied - Techniques employing Ontological Representation for M\&S. In Proceedings of the 2006 Fall Simulation Interoperability Workshop.

Yilmaz, L. 2004. On the Need for Contextualized Introspective Models to Improve Reuse and Composability of Defense Simulations. Journal of Defense Modeling and Simulation 1(3):141-151.

\section{AUTHOR BIOGRAPHIES}

CHARLES TURNITSA is a Ph.D. candidate at the Virginia Modeling Analysis and Simulation Center VMASC - at Old Dominion University. He received his B.S. in Computer Science (1991) from Christopher Newport University (Newport News, Virginia), and his M.S. in Modeling \& Simulation (2006) from ODU. His Ph.D. research under Andreas Tolk focuses on the domain of specifying process activity within dynamic ontological models for $M \& S$ interoperability. His email address is

$<$ cturnitseodu.edu>.

JOSE J. PADILLA is a Post Doctoral Research Associate with the Virginia Modeling, Analysis and Simulation Center - VMASC - at Old Dominion University. He received his Ph.D. in Engineering Management from Old Dominion University. His research interests include knowledge representation, understanding within problem situations, and philosophy of science. His email address is $<$ jpadilladodu.edu>.

ANDREAS TOLK is an Associate Professor of Engineering Management and Systems Engineering at Old Dominion University. He is also affiliated with the Virginia Modeling Analysis and Simulation Center. He holds a M.S. and Ph.D. in Computer Science from the University of the Federal Armed Forces in Munich, Germany. His research focuses on developing mathematical models supporting M\&S composability and system of systems engineering. He is a member of ACM, IEEE, SCS, SISO, MORS, NDIA, and ASEM. His email address is <atol k@odu. edu>. 